\title{
Density Dependent Parametrization Models: Formalism and Applications
}

\author{
S.S. Avancini ${ }^{1}$ and D.P. Menezes ${ }^{1,2}$ \\ ${ }^{1}$ Depto de Física - CFM - Universidade Federal de Santa Catarina \\ Florianópolis - SC - CP. 476 - CEP 88.040 - 900 - Brazil \\ ${ }^{2}$ School of Physics - University of Sydney - NSW 2006 - Australia
}

In this work we derive a formalism to incorporate asymmetry and temperature effects in the Brown-Rho (BR) scaled lagrangian model in a mean field theory. The lagrangian density discussed in this work requires less parameters than the usual models with density dependent couplings. We also present the formalism with the inclusion of the eight lightest baryons, two lightest leptons, $\beta$ equilibrium and charge neutrality in order to apply the BR scaled model to the study of neutron stars. The results are again compared with the ones obtained from another density dependent parametrization model. The role played by the rearrangement term at $\mathrm{T}=0$ for nuclear or neutron star matter and at finite temperature is investigated. The BR scaled model is shown to be a good tool in studies involving density dependent effective masses and in astrophysics applications.

PACS number(s):21.65.+f, 24.10.Jv,26.60.+c,21.30.Fe

\section{INTRODUCTION}

The study of nuclear matter properties at high densities and at finite temperature has become an important problem since a large variety of data, where matter is being tested at extreme conditions of density, pressure and non-zero temperature, are becoming available in the modern experimental facilities which are already operational. Moreover, the constitution of the interior of neutron and protoneutron stars is also a problem which is receiving much attention in the recent literature. The crust of the stars, where density is relatively low, can be adequately described by hadronic models. The correct calculation of the star properties as the radius and the mass depend on the accuracy of the equation of state (EOS) used to describe its matter. We have checked that different models, either with constant or with density dependent meson-nucleon couplings present different features at subnuclear densities of nuclear asymmetric matter by comparing the regions of uniform unstable matter [1]. The parametrizations of these models generally take into account saturation properties of nuclear matter and properties of stable nuclei. Extensions of the models for very asymmetric nuclear matter or to finite temperatures show different behaviors.

Another topic of great interest is the in-medium modification of vector meson properties. It is well known that the hadron masses are much larger than the sum of its constituents. One possible explanation for the large masses is that they may be generated dynamically 22. Spontaneous breaking of chiral symmetry is also related with the hadron masses. At high temperature and/or dense matter this symmetry is expected to be at least partially restored, which modifies the hadron masses and the decay widths [3], 4]. Experimental signature of the in-medium modifications of the vector mesons have been found very recently [5, 6]. In [5] the in medium modifications of the $\omega$ meson were investigated in photoproduction 
experiments and its mass was found to be lowered. In [ 6] the vector masses were verified to decrease by ten percent in medium. Other experimental results were also reported in the same direction 7] and still some experiments have been proposed to detect in-medium modifications in a near future 8$]$. In order to take into account the in-medium modifications of the hadrons, density dependent relativistic models are certainly more useful than models with fixed parameters. In 1991 Brown and Rho (BR) [4] proposed an in-medium scaling law for the masses and coupling constants for effective chiral lagrangians. Our proposal here is to study hadronic properties under extreme conditions using lagrangians which incorporate BR scaling since this has been successfully applied to describe meson properties. As argued in the literature the BR scaling describes the behavior of the light mesons in extreme conditions very accurately. For example the enhancement of dilepton production observed in heavy ion collisions $(\mathrm{S}+\mathrm{Au})$ in the CERES collaboration and $(\mathrm{S}+\mathrm{W})$ in the HELIOS-3 is most economically and beautifully described by a chiral lagrangian with BR-scaled meson masses [9, 10]. The strategy is to assume that the in-medium effective lagrangian has the same structure as in free space accordingly to the QCD constraints but with parameters which are modified in the medium. So, using this approach we obtain an effective theory with density dependent parameters including many-body correlations in a practical framework. It has also been shown that it is possible to obtain a relation between the effective parameters of chiral lagrangians in medium and Landau Fermi Liquid parameters 11]. So, a link between the effective theory of QCD at mean field level and the many-body theory of nuclear matter is achieved. Using this reasoning the authors in [10] proposed an effective Lagrangian whose parameters scale in nuclear medium according to the Brown-Rho (BR) scaling.

We have already shown that one of these density dependent models, to which we refer as TW model 12, 13], originally derived at $T=0$ can be extrapolated to finite temperatures once the thermodynamical consistency remains unaltered [14, 15]. The important range of temperature which is discussed lies between 10 and $150 \mathrm{MeV}$ since the liquid-gas phase transition takes place around $10 \mathrm{MeV}$, the phase transition from hadronic to quarkionic matter around $150 \mathrm{MeV}$ and the relevant temperatures in the cooling of a protoneutron star after a supernova explosion takes place go up to approximately $40 \mathrm{MeV}$

[16]. In this work we discuss another possible application of the formalism we have derived in [14, 15] in order to incorporate temperature effects in the study of lagrangians with BR scaling.

It is well known that the same relativistic models used in order to explain data coming from heavy ion collisions at finite temperature, with appropriate parameter sets, also provide EOS which can be used in the solution of the Tolman-Oppenheimer-Volkoff differential equations [17] for the calculation of stellar properties such as mass, radius and central energy density. Recent measurements of gravitational redshift of spectral lines provided direct constraints on the mass-to-radius ratio [18, 19]. In this second case, however, the interpretation of the absorption features as atomic transition lines is controversial [20, 21]. In recent works [22, 23] we have checked that there are relativistic models which can be accommodated within these constraints. Hence, astrophysical observations can help in the choice of appropriate models to describe hadronic matter. The most common relativistic model used in the description of hadronic matter is the non linear Walecka model 24]. When applied in nuclear astrophysics, this model is normally extended with the inclusion of hyperons, which are expected to appear at high densities. It was shown in 25] that a low effective mass at saturation density makes the model inappropriate once hyperons are included. The inclusion of hyperons makes the scalar meson interaction stronger and hence the proton and neutron effective masses decrease more rapidly with density, acquiring a negative value. As a test of 
the BR scaled model, we also extend it to incorporate the eight lightest baryons and enforce $\beta$ equilibrium plus charge neutrality by accommodating the two lightest leptons as well. The same extension is done within the TW model so that both density dependent models can be compared.

Hence, the present work is organised as follows: in section II the formalism for the BR scaled model and its extension to finite temperature are presented, the results are compared with the ones obtained with other relativist models and a discussion is included. In section III the BR scaled model is modified so that hyperons can be incorporated and $\beta$ equilibrium and charge neutrality are enforced so that an EOS can be obtained and applied to compact stars. In the same section the TW model is also considered so that the results from two density dependent models are compared. In section IV a discussion on the role played by the rearrangement term in different models is presented. Finally, in the last section, the conclusions are drawn.

\section{FORMALISM - EXTENSION TO FINITE TEMPERATURE}

In its simplest version the Lagrangian density reads 10,26 .

$$
\begin{aligned}
\mathcal{L}= & \bar{\psi}\left[\gamma_{\mu}\left(i \partial^{\mu}-g_{v}^{*}(\rho) \omega^{\mu}-g_{\rho}^{*}(\rho) \vec{\tau} \cdot \vec{\rho}^{\mu}\right)-M^{*}(\rho)+g_{s}^{*}(\rho) \phi\right] \psi \\
& +\frac{1}{2}\left(\partial_{\mu} \phi \partial^{\mu} \phi-m_{s}^{* 2}(\rho) \phi^{2}\right)-\frac{1}{4} \Omega_{\mu \nu} \Omega^{\mu \nu}+\frac{1}{2} m_{v}^{* 2}(\rho) \omega_{\mu} \omega^{\mu} \\
& -\frac{1}{4} \vec{\rho}_{\mu \nu} \cdot \vec{\rho}^{\mu \nu}+\frac{1}{2} m_{\rho}^{* 2} \vec{\rho}_{\mu} \cdot \vec{\rho}^{\mu}, .
\end{aligned}
$$

where in the notation of [26] $\psi$ is the nucleon field, $\omega_{\mu}$ the isoscalar vector field, $\phi$ an isoscalar scalar field, $\Omega_{\mu \nu}=\partial_{\mu} \omega_{\nu}-\partial_{\nu} \omega_{\mu}, \overrightarrow{\rho^{\mu}}$ is the vector isovector field, $\vec{\rho}_{\mu \nu}=\partial_{\mu} \vec{\rho}_{\nu}-\partial_{\nu} \vec{\rho}_{\mu}-g_{\rho}^{*}\left(\vec{\rho}_{\mu} \times \vec{\rho}_{\nu}\right), \vec{\tau}$ is the isospin operator and the masses with asterisk are BR-scaled as introduced in [4]:

$$
\frac{M^{*}}{M}=\frac{m_{s}^{*}}{m_{s}}=\frac{m_{v}^{*}}{m_{v}}=\frac{m_{\rho}^{*}}{m_{\rho}}=\Phi(\rho)
$$

The scalings of the vector coupling constants are given by

$$
\frac{g_{s}^{*}}{g_{s}}=\frac{1}{1+x \rho / \rho_{0}}, \quad \frac{g_{v}^{*}}{g_{v}}=\frac{1}{1+z \rho / \rho_{0}}, \quad \frac{g_{\rho}^{*}}{g_{\rho}}=\frac{1}{1+w \rho / \rho_{0}}
$$

where $\rho_{0}$ is the nuclear saturation density. In the original papers [10, 27], $g_{s}^{*}$ was simply taken constant and equal to $g_{s}$ and hence, not density dependent and, with this simple choice a good description of the ground state was obtained. Moreover,

$$
\Phi(\rho)=\frac{1}{1+y \rho / \rho_{0}},
$$

with $y=0.28$ in such a way that $\Phi\left(\rho_{0}\right)=0.78[10]$ and $z$ and $w$ were taken equal or tly greater that $y$. In this work we propose another possible parametrization, based on the works [6, 30], where

$$
\Phi(\rho)=1-y \frac{\rho}{\rho_{0}}
$$

with $y=0.1$ in such a way that a decrease of the meson masses in medium at the saturation point is ten percent, as found experimentally [․ It is also worth mentioning that, as far as we know this is the 
first work where asymmetry is taken into account by the appropriate inclusion of the $\rho$ meson in a BR scaled model. One would bear in mind that we are using the letter $\rho$ for both the meson and for the total baryonic density. As those are common definitions in relativistic models we do not believe it may cause any confusion. One can see that the Lagrangian in (1) is of the form of a Walecka-type Lagrangian and all the finite temperature formalism that we have developed for the density dependent hadron field theory (DDHFT) 14, 15] can be immediately applied to these lagrangians. The thermodynamics of effective lagrangians with BR scaling has been studied in [27] for zero temperature. The study of the validity of the BR scaling hypothesis for the non-zero temperature case is demonstrated in what follows. From the Euler-Lagrange equations we obtain the field equations of motion in the mean field approximation for infinite matter, where the meson fields are replaced by their expectation values. In this approximation, the expectation value of the $\sigma, \omega$ and $\rho$ meson fields are called $\phi_{0}, V_{0}$ and $b_{0}$ respectively. The coupled equations read

$$
\begin{aligned}
m_{s}^{* 2} \phi_{0}-g_{s}^{*} \rho_{s} & =0, \\
m_{v}^{* 2} V_{0}-g_{v}^{*} \rho & =0, \\
m_{\rho}^{* 2} b_{0}-\frac{g_{\rho}^{*}}{2} \rho_{3} & =0, \\
{\left[i \gamma^{\mu} \partial_{\mu}-\gamma_{0}\left(g_{v}^{*} V_{0}+g_{\rho}^{*} \tau_{3} b_{0}+\Sigma_{0}^{R}\right)-\left(M^{*}-g_{s}^{*} \phi_{0}\right)\right] \psi } & =0,
\end{aligned}
$$

where the rearrangement term $\Sigma_{0}^{R}$ is given by

$$
\Sigma_{0}^{R}=-m_{v}^{*} V_{0}^{2} \frac{\partial m_{v}^{*}}{\partial \rho}+\rho V_{0} \frac{\partial g_{v}^{*}}{\partial \rho}-m_{\rho}^{*} b_{0}^{2} \frac{\partial m_{\rho}^{*}}{\partial \rho}+\rho_{3} \frac{b_{0}}{2} \frac{\partial g_{\rho^{*}}}{\partial \rho}+m_{s}^{*} \phi_{0}^{2} \frac{\partial m_{s}^{*}}{\partial \rho}+\rho_{s} \frac{\partial M^{*}}{\partial \rho}-\rho_{s} \phi_{0} \frac{\partial g_{s}^{*}}{\partial \rho}
$$

and the scalar and baryonic densities are defined as

$$
\begin{aligned}
\rho_{s} & =\langle\bar{\psi} \psi\rangle, \\
\rho & =\left\langle\bar{\psi} \gamma^{0} \psi\right\rangle, \\
\rho_{3} & =2\left\langle\bar{\psi} \gamma^{0} \tau_{3} \psi\right\rangle .
\end{aligned}
$$

Notice that if the original parametrization for the BR-scaled model is used, $g_{s}^{*}=g_{s}$ is a constant and the last term of the rearrangement vanishes. In the following discussion we consider nuclear matter in the the mean-field approximation. Due to translational and rotational invariance the lagrangian density reduces to

$$
\begin{aligned}
\mathcal{L}_{M F T}= & \bar{\psi}\left[i \gamma_{\mu} \partial^{\mu}-\gamma_{0} g_{v}^{*} V_{0}-\gamma_{0} g_{\rho}^{*} \tau_{3} b_{0}-\left(M^{*}-g_{s}^{*} \phi_{0}\right)\right] \psi \\
& -\frac{1}{2} m_{s}^{* 2} \phi_{0}^{2}+\frac{1}{2} m_{v}^{* 2} V_{0}^{2}+\frac{1}{2} m_{\rho}^{* 2} b_{0}^{2}
\end{aligned}
$$

where $\tau_{3}= \pm 1 / 2$ for protons and neutrons respectively. The conserved energy-momentum tensor can be derived in the usual fashion [24]:

$$
\mathcal{T}_{M F T}^{\mu \nu}=\bar{\psi} i \gamma^{\mu} \partial^{\nu} \psi+g^{\mu \nu}\left[\frac{1}{2} m_{s}^{* 2} \phi_{0}^{2}-\frac{1}{2} m_{v}^{* 2} V_{0}^{2}-\frac{1}{2} m_{\rho}^{* 2} b_{0}^{2}+\bar{\psi} \gamma_{0} \Sigma_{0}^{R} \psi\right]
$$

Note that the rearrangement term included above and defined in eq.(10) assures the energy-momentum conservation, i.e., $\partial_{\mu} \mathcal{T}^{\mu \nu}=0$. From the energy-momentum tensor one easily obtains the hamiltonian 
operator:

$$
\begin{aligned}
\mathcal{H}_{M F T}=\int d^{3} x \mathcal{T}_{M F T}^{00}= & \int d^{3} x \psi^{\dagger}\left(-i \vec{\alpha} \cdot \nabla+\beta m_{L}^{*}+g_{v}^{*} V_{0}+g_{\rho}^{*} \tau_{3} b_{0}+\Sigma_{0}^{R}\right) \psi \\
& +V\left(\frac{1}{2} m_{s}^{* 2} \phi_{0}^{2}-\frac{1}{2} m_{v}^{* 2} V_{0}^{2}-\frac{1}{2} m_{\rho}^{* 2} b_{0}^{2}\right)
\end{aligned}
$$

where

$$
m_{L}^{*}=M^{*}-g_{s}^{*} \phi_{0}
$$

is identified as the effective nucleon Landau mass and $V$ is the volume of the system. Notice that the energy density does not carry the rearrangement term because it cancels out in a mean field approximation:

$$
\mathcal{E}=2 \sum_{i=p, n} \int \frac{d^{3} p}{(2 \pi)^{3}} \sqrt{\mathbf{p}^{2}+m_{L}^{* 2}}\left(f_{i+}+f_{i-}\right)+\frac{m_{s}^{* 2}}{2} \phi_{0}^{2}+\frac{m_{v}^{* 2}}{2} V_{0}^{2}+\frac{m_{\rho}^{2}}{2} b_{0}^{2},
$$

where $f_{i+}$ and $f_{i-}$ are the distribution functions for particles and anti-particles respectively and are calculated next. Following the notation in [28], the thermodynamic potential can be written as

$$
\Omega=\mathcal{E}-T \mathcal{S}-\mu_{p} \rho_{p}-\mu_{n} \rho_{n}
$$

where $\mathcal{S}$ is the entropy of a classical Fermi gas, $T$ is the temperature, $\mu_{p}\left(\mu_{n}\right)$ is the proton (neutron) chemical potential and $\rho_{p}$ and $\rho_{n}$ are respectively the proton and neutron densities, calculated in such a way that $\rho=\rho_{p}+\rho_{n}$. We have

$$
\rho_{i}=2 \int \frac{d^{3} p}{(2 \pi)^{3}}\left(f_{i+}-f_{i-}\right), \quad i=p, n,
$$

where the distribution functions $f_{i+}$ and $f_{i-}$ for particles and anti-particles have to be derived in order to make the thermodynamic potential stationary for a system in equilibrium. After straightforward substitutions, eq. (19) becomes

$$
\begin{aligned}
\Omega= & 2 \sum_{i=p, n} \int \frac{d^{3} p}{(2 \pi)^{3}} \sqrt{\mathbf{p}^{2}+m_{L}^{* 2}}\left(f_{i+}+f_{i-}\right)+\frac{m_{s}^{* 2}}{2} \phi_{0}^{2}+\frac{m_{v}^{* 2}}{2} V_{0}^{2}+\frac{m_{\rho}^{* 2}}{2} b_{0}^{2} \\
& +2 T \sum_{i=p, n} \int \frac{d^{3} p}{(2 \pi)^{3}}\left(f_{i+} \ln \left(\frac{f_{i+}}{1-f_{i+}}\right)+\ln \left(1-f_{i+}\right)+f_{i-} \ln \left(\frac{f_{i-}}{1-f_{i-}}\right)+\ln \left(1-f_{i-}\right)\right) \\
& -2 \sum_{i=p, n} \int \frac{d^{3} p}{(2 \pi)^{3}} \mu_{i}\left(f_{i+}-f_{i-}\right) .
\end{aligned}
$$

For a complete demonstration of the above shown expressions obtained in a Thomas-Fermi approximation for the non-linear Walecka model, please refer to 28]. At this point, eq. 21] is minimized in terms of the distribution functions for fixed meson fields, i.e.,

$$
\left.\frac{\partial \Omega}{\partial f_{i+}}\right|_{f_{i-}, f_{j \pm}, \phi_{0}, V_{0}, b_{0}}=0 \quad i \neq j
$$

For the particle distribution function, the above calculation yields

$$
E^{*}(\mathbf{p})+\Sigma_{0}^{R}-\mu_{i}+g_{v}^{*} V_{0}+\frac{g_{\rho}^{*}}{2} b_{0}=-T \ln \left(\frac{f_{i+}}{1-f_{i+}}\right)
$$


where $E^{*}(\mathbf{p})=\sqrt{\mathbf{p}^{2}+m_{L}^{* 2}}$. A similar equation, with a sign difference is obtained for the anti-particle distribution function. It is important to point out that the fields $\phi_{0}, V_{0}$ and $b_{0}$ depend on the distribution function which appear in the definition of $\rho_{s}, \rho$ and $\rho_{3}$ and hence, the whole calculation is performed self-consistently. The effective chemical potentials are then defined as

$$
\begin{aligned}
& \nu_{p}=\mu_{p}-g_{v}^{*} V_{0}-\frac{g_{\rho}^{*}}{2} b_{0}-\Sigma_{0}^{R}, \\
& \nu_{n}=\mu_{n}-g_{v}^{*} V_{0}+\frac{g_{\rho}^{*}}{2} b_{0}-\Sigma_{0}^{R}
\end{aligned}
$$

and the following equations for the distribution functions can be written:

$$
f_{i \pm}=\frac{1}{1+\exp \left[\left(E^{*}(\mathbf{p}) \mp \nu_{i}\right) / T\right]}, \quad i=p, n .
$$

In the above calculation we have used

$$
\rho_{s}=2 \sum_{i=p, n} \int \frac{d^{3} p}{(2 \pi)^{3}} \frac{m_{L}^{*}}{E^{*}(\mathbf{p})}\left(f_{i+}+f_{i-}\right),
$$

and $\rho_{3}=\rho_{p}-\rho_{n}$. Within the Thomas-Fermi approach the pressure becomes

$$
\begin{aligned}
P= & \frac{1}{3 \pi^{2}} \sum_{i=p, n} \int d p \frac{\mathbf{p}^{4}}{\sqrt{\mathbf{p}^{2}+M^{* 2}}}\left(f_{i+}+f_{i-}\right) \\
& -\frac{m_{s}^{* 2}}{2} \phi_{0}^{2}+\frac{m_{v}^{* 2}}{2} V_{0}^{2}+\frac{m_{\rho}^{* 2}}{2} b_{0}^{2}+\Sigma_{0}^{R} \rho .
\end{aligned}
$$

It is worth mentioning that the thermodynamical consistency which requires the equality of the pressure calculated from the thermodynamical definition and from the energy-momentum tensor, discussed in [15], is also obeyed by the temperature dependent Brown-Rho scaled model.

\section{A. Discussions on the BR-scaled model for nuclear matter}

At this point, the parameters used in the BR method have to be fixed. Through out this paper the nucleon mass will be $M=939 \mathrm{MeV}$, the $\omega$ meson mass $m_{v}=783 \mathrm{MeV}$ and the $\rho$ meson mass $m_{\rho}=763$

$\mathrm{MeV}$. Three different sets are used in [27]. In what follows we use the parameter set called S3 in [27] and define another one which we call MA, whose bulk properties are more similar to the NL3 [29] parameter set but with a larger effective mass at nuclear saturation density. While S3 is a parametrization for the original $\mathrm{BR}$ scaled model given by equation (4), MA is a parametrization for the new scaling, given in

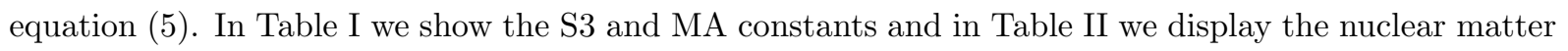
bulk properties described by the different models used in this work. It is important to point out that the value for the saturation density (vide* in Table II) was not given in [10]. For the saturation density value shown in Table \ the compressibility is slightly different from what is stated in 10$](260 \mathrm{MeV})$. This is probably a consequence of the fact that the authors in [10] have not included the rearrangement term in their calculations. Notice that we distinguish the $M^{*}$ from the $m_{L}^{*}$ values. In the BR scaled models it is the Landau mass that should be identified with the nucleon effective mass determined by the QCD sum rule [10] and its value should lie in between $0.55 \mathrm{M}$ and $0.75 \mathrm{M}$. 
TABLE I: Parameter sets for the Lagrangian (1)

\begin{tabular}{|c|c|c|c|c|c|c|c|}
\hline Set & $m_{s}$ & $g_{s}$ & $g_{v}$ & $g_{\rho}$ & $x$ & $z$ & $w$ \\
\hline S3 & 700 & 5.30 & 15.2 & 7.97 & - & 0.31 & 0.31 \\
MA & 500 & 7.05 & 12.006 & 8.761 & 0.37 & 0.15 & 0.15 \\
\hline
\end{tabular}

TABLE II: Nuclear matter properties

\begin{tabular}{|c|c|c|c|c|c|c|}
\hline & NL3 [29] & TM1 [33] & GL [34] & TW [12] & S3 [27] & MA \\
\hline$B / A(\mathrm{MeV})$ & 16.3 & 16.3 & 15.95 & 16.3 & 16.1 & 16.3 \\
\hline$\rho_{0}\left(\mathrm{fm}^{-3}\right)$ & 0.148 & 0.145 & 0.145 & 0.148 & $0.155\left(^{*}\right)$ & 0.148 \\
\hline$K(\mathrm{MeV})$ & 272 & 281 & 285 & 240 & 269 & 258 \\
\hline $\mathcal{E}_{\text {sym. }}(\mathrm{MeV})$ & 37.4 & 36.9 & 36.8 & 32.0 & 32.0 & 32.0 \\
\hline$M^{*} / M$ & 0.60 & 0.63 & 0.77 & 0.56 & 0.78 & 0.9 \\
\hline$m_{L}^{*} / M$ & - & - & - & - & 0.68 & 0.748 \\
\hline
\end{tabular}

In figure 1 we plot the dependence of the meson masses with the density for the S3 and MA parameter sets. This is an important consequence of this model, since the reduction of the meson masses in medium is an expected result [30]. As stated in the Introduction, this behavior is related with the restoration of the chiral symmetry and experiments with the spectrometer HADES at GSI will also be measuring this effect soon. If the S3 parametrization is used, the effective masses of all mesons decrease by $22 \%$ up to the saturation density while if the MA parametrization is used, the decrease is forced to be just $10 \%$, as found in 66]. In figure 2 we display the behavior of the coupling constants. Although both $g_{v}^{*}$ 's are quite different at subsaturation densities, they tend to achieve reasonably close values at larger densities. $g_{\rho}^{*}$, on the other hand, presents quite a similar behavior in both models and $g_{s}^{*}$ only changes with density within the MA framework. In figure 3 we show the ratios $\left(g_{i}^{*} / m_{i}^{*}\right)^{2}$, with $i=s, v, \rho$ which are quantities always present in nuclear matter calculations. One can see that the ratios are very small, the ratio involving the $\omega$ meson being the largest in both parametrizations. One should bear in mind that, as stated earlier, within the original version of the BR scaled model, the scalar coupling constant does not vary with the density. For the MA set, on the other hand, the ratio $\left(g_{s}^{*} / m_{s}^{*}\right)^{2}$ tends to zero at $\simeq 2 \rho_{0}$.

In figure 4 we show the binding energy in terms of the baryon density for different models for $T=0$ and $T=40 \mathrm{MeV}$. For the sake of comparison with other models, we have chosen one model with constant couplings (NL3) and another one with density dependent couplings (TW). At $T=0$, the TW model is the softest one and the NL3 the hardest, the two curves obtained with the BR scaled parametrizations interpolating between the other models. One can see that the temperature does not alter the softness (hardness) of the EOS considered. The hardest and the softest ones at $T=0$ remain so at a higher temperature.

In figure 5 the pressure versus the baryon density is displayed for symmetric nuclear matter $\left(y_{p}=0.5\right)$ and for very asymmetric matter $\left(y_{p}=0.1\right)$, where $y_{p}=\rho_{p} / \rho$ is the proton fraction. The isospin is a quantity which influences the softness (hardness) of the EOS, but one can see that the asymmetry seems not to affect the displayed EOSs.

In figure 6 we show how the temperature affects the binding energy of the S3 and MA models for symmetric matter. The behavior is the same one encountered in 14], i.e., the minimum shifts to higher 
densities with the increase of the temperature and moves from a negative to a positive value. This seems to be a natural consequence of the increase in the temperature of the system.

Another quantity of interest is the nuclear bulk symmetry energy discussed in 31]. For symmetric nuclear matter at $\mathrm{T}=0$ it is defined as

$$
\mathcal{E}_{\text {sym }}=\frac{P_{F}^{2}}{6 E^{*}\left(P_{F}\right)}+\frac{g_{\rho}^{* 2}}{8 m_{\rho}^{* 2}} \rho,
$$

with $P_{F}=\left(1.5 \pi^{2} \rho\right)^{1 / 3}$. The value and behavior of the symmetry energy at densities larger than nuclear saturation density are still not well established. This quantity is important in studies involving neutron skins, radioactive nuclei and neutron stars. In general, relativistic and non-relativistic models give different predictions for the symmetry energy. The results of this quantity for different models are also discussed in the present work and the values at saturation density are shown in Table In figure 7 we plot the symmetry energy for the BR scaled models, NL3 and TW. In S3, MA and TW the symmetry energy at saturation density is the same. The three curves do not cross in the same point because the saturation densities are not the same (see Table $\amalg$. Notice that although S3 and MA present a lower symmetry energy at subsaturation densities, they interpolate between NL3 and TW at larger densities. The value of $32 \mathrm{MeV}$ that we have chosen for the symmetry energy in order to fix the $g_{\rho}$ coupling is lower than the ones found in most relativistic models (between 35 and $42 \mathrm{MeV}$ ) and approaches the values obtained in non-relativistic models (between 28 and $38 \mathrm{MeV}$ ). Notice that the choice of parameters is not arbitrary. They are chosen in order to reproduce the nuclear bulk properties of Table Moreover, they also have to give the correct value of the spin-orbit splitting strength. Work in the direction of calculating this quantity in finite nuclei is in progress, which may require small changes in the calculated parameter sets.

\section{APPLICATION TO COMPACT STARS}

From the results shown in the previous section we could see that the BR scaled models show quite a different behavior from the NL3 and TW models at high densities. In what follows we intend to investigate which are the consequences of using the BR scaled model in the description of neutron star matter. The behavior of the EOS at high densities is responsible for the determination of the maximum mass of the star. In order to apply the BR scaled density dependent model to compact stellar objects, it is important to allow for the inclusion of the eight lightest baryons (nucleons, $\Lambda, \Sigma^{0}, \Sigma^{ \pm}, \Xi^{-}$and $\Xi^{0}$ ) as well as the two lightest leptons $\left(e^{-}\right.$and $\mu$ ). The baryons have to be considered since their masses are such that their presence is already possible at the neutron stars high densities. The leptons, on the other

hand, play a decisive role in ensuring charge neutrality and $\beta$ equilibrium. As seen in equation (24) the rearrangement term which appears due to the density dependent couplings alters the chemical potentials of the particles in the system and hence, the $\beta$ equilibrium conditions are somewhat different as compared with the usual relativistic models. In what follows we show the formalism developed for the BR scaled model in neutron stars and also for the TW model, so that two density dependent parametrization models can be compared. 


\section{A. Considering $\beta$ equilibrium within the BR-scaled model}

For our purposes of testing the BR scaled model at high densities, which are present in neutron stars, we shall restrict ourselves to the $T=0$ case. Of course, the extension to finite temperature is trivial and can be done following the steps of section II. Actually in calculations involving protoneutron stars or stars with fixed entropy and trapped neutrinos, the extension has to be done. Equation (1) is then modified in order to accommodate these new particles

$$
\begin{aligned}
\mathcal{L}= & \sum_{B} \bar{\psi}_{B}\left[\gamma_{\mu}\left(i \partial^{\mu}-g_{v B}^{*}(\rho) \omega^{\mu}-g_{\rho B}^{*}(\rho) \vec{\tau} \cdot \vec{\rho}^{\mu}\right)-M_{B}^{*}(\rho)+g_{s B}^{*}(\rho) \phi\right] \psi_{B} \\
& +\frac{1}{2}\left(\partial_{\mu} \phi \partial^{\mu} \phi-m_{s}^{* 2}(\rho) \phi^{2}\right)-\frac{1}{4} \Omega_{\mu \nu} \Omega^{\mu \nu}+\frac{1}{2} m_{v}^{* 2}(\rho) \omega_{\mu} \omega^{\mu} \\
& -\frac{1}{4} \vec{\rho}_{\mu \nu} \cdot \vec{\rho}^{\mu \nu}+\frac{1}{2} m_{\rho}^{* 2} \vec{\rho}_{\mu} \cdot \vec{\rho}^{\mu}+\sum_{l} \bar{\psi}_{l}\left(i \gamma_{\mu} \partial^{\mu}-m_{l}\right) \psi_{l} .
\end{aligned}
$$

where the meson field operators represent the same mesons as in eq. (11), $\psi_{B}$ now represents each of the eight baryons, $l$ describes the two leptons whose masses are respectively $m_{e}=0.511 \mathrm{MeV}$ and $m_{\mu}=106.55 \mathrm{MeV}$ and the masses with asterisk are again BR-scaled as in eq. (2), the 8 baryons of the octet also obeying the same scaling law, i.e.,

$$
\frac{M_{B}^{*}}{M_{B}}=\Phi(\rho)
$$

The baryon meson couplings are defined as $g_{s B}^{*}=x_{s B} g_{s}^{*}, \quad g_{v B}^{*}=x_{v B} g_{v}^{*}, \quad g_{\rho B}^{*}=x_{\rho B} g_{\rho}^{*}$ and $x_{s B}, x_{v B}$ and $x_{\rho B}$ are equal to 1 for the nucleons and may have different values for the hyperons. Again the meson fields are obtained in the same way as in section II and they now read:

$$
\begin{aligned}
m_{s}^{* 2} \phi_{0}-\sum_{B} g_{s B}^{*} \rho_{s B} & =0, \\
m_{v}^{* 2} V_{0}-\sum_{B} g_{v B}^{*} \rho_{B} & =0, \\
m_{\rho}^{* 2} b_{0}-\sum_{B} g_{\rho B}^{*} \tau_{3 B} \rho_{B} & =0, \\
{\left[i \gamma^{\mu} \partial_{\mu}-\gamma_{0}\left(g_{v B}^{*} V_{0}+g_{\rho B}^{*} \tau_{3 B} b_{0}+\Sigma_{0 B R}^{R}\right)-\left(M_{B}^{*}-g_{s B}^{*} \phi_{0}\right)\right] \psi } & =0,
\end{aligned}
$$

where the term $\Sigma_{0}^{R}$ BR is now changed and is given by

$$
\begin{gathered}
\Sigma_{0 B R}^{R}=-m_{v}^{*} V_{0}^{2} \frac{\partial m_{v}^{*}}{\partial \rho}+\sum_{B} \rho_{B} V_{0} \frac{\partial g_{v B}^{*}}{\partial \rho}-m_{\rho}^{*} b_{0}^{2} \frac{\partial m_{\rho}^{*}}{\partial \rho}+\sum_{B} \tau_{3 B} \rho_{B} b_{0} \frac{\partial g_{\rho B}^{*}}{\partial \rho} \\
+m_{s}^{*} \phi_{0}^{2} \frac{\partial m_{s}^{*}}{\partial \rho}+\sum_{B} \rho_{s B} \frac{\partial M_{B}^{*}}{\partial \rho}-\rho_{s B} \phi_{0} \frac{\partial g_{s B}^{*}}{\partial \rho}
\end{gathered}
$$

$\tau_{3 B}$ is the isospin projection of each baryon and the scalar and baryonic densities are

$$
\begin{aligned}
\rho_{s B} & =\frac{1}{\pi^{2}} \int p^{2} d p \frac{m_{L B}^{*}}{E_{B}^{*}}, \\
\rho_{B} & =\frac{k_{F B}^{3}}{3 \pi^{2}},
\end{aligned}
$$


with

$$
\begin{aligned}
m_{L B}^{*} & =M_{B}^{*}-g_{s B}^{*} \phi_{0} \\
E_{B}^{*} & =\sqrt{\mathbf{p}^{2}+m_{L B}^{*}{ }^{2}} \\
\nu_{B} & =\mu_{B}-g_{v B}^{*} V_{0}-g_{\rho B}^{*} \tau_{3 B} b_{0}-\Sigma_{0 B R}^{R} \\
& =\sqrt{k_{F B}^{2}+m_{L B}^{*}{ }^{2}} .
\end{aligned}
$$

The equation of state, necessary for the description of the stellar matter can now be obtained. The energy density and the pressure density are given respectively by

$$
\mathcal{E}=\frac{1}{\pi^{2}} \sum_{B} \int_{0}^{k_{F B}} p^{2} d p E_{B}^{*}+\frac{m_{s}^{* 2}}{2} \phi_{0}^{2}+\frac{m_{v}^{* 2}}{2} V_{0}^{2}+\frac{m_{\rho}^{* 2}}{2} b_{0}^{2}+\frac{1}{\pi^{2}} \sum_{l} \int_{0}^{k_{F l}} p^{2} d p E_{l}
$$

and

$$
P=\frac{1}{3 \pi^{2}} \sum_{B} \int_{0}^{k_{F B}} \frac{p^{4} d p}{E_{B}^{*}}-\frac{m_{s}^{* 2}}{2} \phi_{0}^{2}+\frac{m_{v}^{* 2}}{2} V_{0}^{2}+\frac{m_{\rho}^{* 2}}{2} b_{0}^{2}+\left(\sum_{B} \rho_{B}\right) \Sigma_{0 B R}^{R}+\frac{1}{3 \pi^{2}} \sum_{l} \int_{0}^{k_{F l}} \frac{p^{4} d p}{E_{l}},
$$

where $E_{l}=\sqrt{\mathbf{p}^{2}+m_{l}^{2}}, \rho_{l}=\frac{k_{F l}^{3}}{3 \pi^{2}}$ and $k_{F l}=\sqrt{\mu_{l}^{2}-m_{l}^{2}}, \mu_{l}$ being the chemical potential of the lepton $l$. Notice that, as far as the leptons do not exchange mesons with the baryons nor with themselves, they were introduced as free Fermi gases. The weak interaction between leptons and hadrons is taken into account through the constraints of charge neutrality and $\beta$ equilibrium given respectively by:

$$
\sum_{B} q_{B}^{e} \rho_{B}+\sum_{l} q_{l}^{e} \rho_{l}=0
$$

where $q_{B}^{e}$ is the electric charge of baryon $B, q_{l}^{e}$ is the electric charge of lepton $l$ and

$$
\mu_{B}=\mu_{n}-q_{B}^{e} \mu_{e}
$$

\section{B. Considering $\beta$ equilibrium within the TW model}

In order to make a comparison with the density dependent BR scaled model, we next make some considerations about the TW model [12], originally derived at $T=0$ and which has also been extrapolated to finite temperatures 14,15 . In what follows we write the most important formulae for the TW model once the lightest baryon octet and the lightest leptons are included and charge neutrality and $\beta$ equilibrium are enforced. In reference [32] a similar approach was developed and two different models were discussed. In the first of them the couplings depend on the total baryonic density, as done in the BR scaled approach shown in the last subsection and also next in the present work. In the second model the couplings depend only on the proton plus neutron densities. The authors of reference 32] showed that an examination of the neutron star properties favored the first model. Notice that we next redefine many of the previously defined quantities, as the baryon effective mass, baryon chemical potentials, etc. The new equations should not be mixed up with the equations given in the previous subsections although we have kept the same notation. 
We start from the lagrangian density

$$
\begin{aligned}
\mathcal{L}= & \sum_{B} \bar{\psi}_{B}\left[\gamma_{\mu}\left(i \partial^{\mu}-\Gamma_{v B} \omega^{\mu}-\Gamma_{\rho B} \vec{\tau} \cdot \vec{\rho}^{\mu}\right)-\left(M-\Gamma_{s B} \phi\right)\right] \psi_{B} \\
& +\frac{1}{2}\left(\partial_{\mu} \phi \partial^{\mu} \phi-m_{s}^{2} \phi^{2}\right)-\frac{1}{4} \Omega_{\mu \nu} \Omega^{\mu \nu}+\frac{1}{2} m_{v}^{2} \omega_{\mu} \omega^{\mu} \\
& -\frac{1}{4} \vec{\rho}_{\mu \nu} \cdot \vec{\rho}^{\mu \nu}+\frac{1}{2} m_{\rho}^{2} \vec{\rho}_{\mu} \cdot \vec{\rho}^{\mu}+\sum_{l} \bar{\psi}_{l}\left(i \gamma_{\mu} \partial^{\mu}-m_{l}\right) \psi_{l}
\end{aligned}
$$

with all the definitions for the fields given after eq. (29) still holding. $\Gamma_{i B}$ and $m_{i}$ are respectively the couplings of the mesons $i=s, v, \rho$ with the hyperons and their masses. In this model the set of constants is defined by $\Gamma_{s B}=x_{s B} \Gamma_{s}, \quad \Gamma_{v B}=x_{v B} \Gamma_{v}, \quad \Gamma_{\rho B}=x_{\rho B} \Gamma_{\rho}$ and as in subsection III A, $x_{s B}, x_{v B}$ and $x_{\rho B}$ are equal to 1 for the nucleons and can acquire different values for the hyperons. $\Gamma_{s}, \Gamma_{v}$ and $\Gamma_{\rho}$ are the nucleon-meson coupling constants which are adjusted in order to reproduce some of the nuclear matter bulk properties, using the following parametrization:

$$
\Gamma_{i}(\rho)=\Gamma_{i}\left(\rho_{0}\right) f_{i}(x), \quad i=s, v
$$

with

$$
f_{i}(x)=a_{i} \frac{1+b_{i}\left(x+d_{i}\right)^{2}}{1+c_{i}\left(x+d_{i}\right)^{2}}
$$

where $x=\rho / \rho_{0}$ and

$$
\Gamma_{\rho}(\rho)=\Gamma_{\rho}\left(\rho_{0}\right) \exp \left[-a_{\rho}(x-1)\right]
$$

with the values of the parameters $m_{j}, \Gamma_{j}, a_{j}, b_{i}, c_{i}$ and $d_{i}, j=s, v, \rho$ given in [12]. The nucleon, $\omega$ and $\rho$ meson masses are taken as in the BR scaled model. The scalar meson mass $m_{s}$ is $500 \mathrm{MeV}$. Other possibilities for these parameters are also found in the literature [35]. The meson and baryon coupled equations for the fields read:

$$
\begin{aligned}
m_{s}^{2} \phi_{0}-\sum_{B} \Gamma_{s B} \rho_{s B} & =0, \\
m_{v}^{2} V_{0}-\sum_{B} \Gamma_{v B} \rho_{B} & =0 \\
m_{\rho}^{2} b_{0}-\sum_{B} \Gamma_{\rho B} \tau_{3 B} \rho_{B} & =0, \\
{\left[i \gamma^{\mu} \partial_{\mu}-\gamma_{0}\left(\Gamma_{v B} V_{0}+\Gamma_{\rho B} \tau_{3 B} b_{0}+\Sigma_{0 T W}^{R}\right)-M_{B}^{*}\right] \psi } & =0,
\end{aligned}
$$

where the term $\Sigma_{0 T W}^{R}$ is given by

$$
\Sigma_{0 T W}^{R}=\sum_{B}\left[\frac{\partial \Gamma_{v B}}{\partial \rho} \rho_{B} V_{0}+\frac{\partial \Gamma_{\rho B}}{\partial \rho} \tau_{3 b} \rho_{B} b_{0}-\frac{\partial \Gamma_{s B}}{\partial \rho} \rho_{s B} \phi_{0},\right]
$$

and the scalar and baryonic densities are defined as

$$
\begin{aligned}
\rho_{s B} & =\frac{1}{\pi^{2}} \int p^{2} d p \frac{M_{B}^{*}}{E_{B}^{*}}, \\
\rho_{B} & =\frac{K_{F B}^{3}}{3 \pi^{2}}
\end{aligned}
$$


with

$$
\begin{aligned}
M_{B}^{*} & =M_{B}-\Gamma_{s B} \phi_{0} \\
E_{B}^{*} & =\sqrt{\mathbf{p}^{2}+M_{B}^{* 2}} .
\end{aligned}
$$

Notice that the rearrangement term shown in equation (54) is the same one shown in equation (18) of 32], once the delta and the strange mesons are excluded from their calculation. The effective chemical potentials are then defined as

$$
\nu_{B}=\mu_{B}-\Gamma_{v B} V_{0}-\Gamma_{\rho B} \tau_{3 B} b_{0}-\Sigma_{0 T W}^{R}=\sqrt{k_{F B}^{2}+M_{B}^{* 2}}
$$

The conditions for $\beta$ equilibrium and charge neutrality are again the same ones as in subsection III A, given by eqs. (44) and (45). The final expressions for the energy density and pressure become respectively:

$$
\mathcal{E}=\frac{1}{\pi^{2}} \sum_{B} \int_{0}^{k_{F B}} p^{2} d p E_{B}^{*}+\frac{m_{s}^{2}}{2} \phi_{0}^{2}+\frac{m_{v}^{2}}{2} V_{0}^{2}+\frac{m_{\rho}^{2}}{2} b_{0}^{2}+\frac{1}{\pi^{2}} \sum_{l} \int_{0}^{k_{F l}} p^{2} d p E_{l}
$$

and

$$
P=\frac{1}{3 \pi^{2}} \sum_{B} \int_{0}^{k_{F B}} \frac{p^{4} d p}{E_{B}^{*}}-\frac{m_{s}^{2}}{2} \phi_{0}^{2}+\frac{m_{v}^{2}}{2} V_{0}^{2}+\frac{m_{\rho}^{2}}{2} b_{0}^{2}+\left(\sum_{B} \rho_{B}\right) \Sigma_{0 T W}^{R}+\frac{1}{3 \pi^{2}} \sum_{l} \int_{0}^{k_{F l}} \frac{p^{4} d p}{E_{l}} .
$$

\section{Discussions on the compact star properties}

Although NL3 29] and TM1 33] are the most common parametrizations of the NLWM for nuclear matter and finite nuclei studies, it is well known that they are just adequate for the description of neutron star properties if only protons, neutrons and leptons are considered as possible constituents [25]. The inclusion of hyperons softens the EOS, but also makes the baryon effective masses decrease too fast and a good convergence can only be obtained at relatively low densities. For this reason, whenever hyperons are considered in the present work, we shall make comparisons with the GL 34] parametrization of the NLWM, where the above mentioned problem does not exist. It is our aim also to verify whether this problem is present in the TW and BR scaled model.

At this point the meson-hyperon couplings have to be fixed. Several possibilities are discussed in the literature [34, 36]. According to [34, 37] the hyperon couplings constrained by the binding of the $\Lambda$ hyperon in nuclear matter, hypernuclear levels and neutron star masses yields $x_{s B}=0.7$ and $x_{v B}=x_{\rho B}=0.783$ and the couplings to the $\Sigma$ and $\Xi$ are equal to those of the $\Lambda$ hyperon. Another possibility is to take $x_{s B}=x_{v B}=x_{\rho B}=\sqrt{2 / 3}$ as in [36, 38, 39]. This choice is based on quark counting arguments. The universal coupling, with $x_{s B}=x_{v B}=x_{\rho B}=1$ has also been used [40]. From [23, 41] it can be verified that the compact star properties depend on the choice of these parameters. As our aim in the present work is to compare results obtained from different models and the correct choice is still not well established, we have used the simple universal coupling in what follows.

In figure 8 we show the particle population for the NLWM with the GL parametrization, for the TW and the BR scaled model either with nucleons only or with the 8 baryons. The particle fraction is defined as $Y_{i}=\rho_{i} / \rho, i=8$ baryons and 2 leptons. We have again chosen two possibilities for the BR scaled parameters, namely S3 and MA. If just protons and neutrons are included, the TW model presents a 
slight decrease in protons and consequently slight increase in neutrons and the MA model shows the opposite behavior as compared with the GL parametrization. If hyperons are considered the results are all quite different, as a consequence of the different EOS shown in figure 9 One can clearly see that all surviving particles tend to the same amount in the BR scaled model, probably a consequence of the enforced scaling law, a feature which happens earlier within S3 than with MA. From figure 9 one can see that in both cases, the TW EOS is the softest one and the MA the hardest. S3 and GL interpolate between the other two EOS. For the present choice of parameters, the TW model also stops converging at a too low density for astrophysical studies. The consequences of this fact will be discussed next.

Once the EOS are obtained, we solve the Tolman-Oppenheimer-Volkoff equations [17] in order to obtain the stellar properties. They read

$$
\begin{gathered}
\frac{d P}{d r}=-\frac{G}{r} \frac{[\mathcal{E}+P]\left[M+4 \pi r^{3} P\right]}{(r-2 G M)}, \\
\frac{d M}{d r}=4 \pi r^{2} \mathcal{E}
\end{gathered}
$$

with $G$ as the gravitational constant and $M(r)$ as the enclosed gravitational mass. We have used $c=1$. Given an EOS, these equations can be integrated from the origin as an initial value problem for a given choice of the central energy density, $\left(\varepsilon_{0}\right)$. The value of $r(=R)$, where the pressure vanishes defines the surface of the star. In Table III we display the results for the stars with the maximum gravitational mass, the maximum baryonic mass, their radii and central energy density for each of the EOS discussed in the present work. One can see that, if only nucleons are considered, the TW model presents the lowest maximum mass and the smallest radius with a consequent very large central energy density. If hyperons are included the obtained result for the TW model is just shown for completeness because it is unrealistic once the maximum mass was not achieved since the program failed to converge at high densities. The results obtained for the maximum masses with the BR scaled models are somewhat larger than with the GL model, but still in the expected range of values. Different results can be obtained with a different choice of the $x_{s B}, x_{v B}$ and $x_{\rho B}$ constants. Once hyperons are included, the EOSs always become softer with a consequent lower value for the maximum stellar masses and radii and larger central energy density.

\begin{tabular}{|c|c|c|c|c|c|}
\hline type & hadron model & $M_{\max }\left(M_{\odot}\right)$ & $M_{b \max }\left(M_{\odot}\right)$ & $R(\mathrm{Km})$ & $\varepsilon_{0}\left(\mathrm{fm}^{-4}\right)$ \\
\hline np & GL & 2.40 & 2.89 & 12.19 & 5.43 \\
\hline $\mathrm{np}+$ hyperons & GL & 2.18 & 2.56 & 11.35 & 6.34 \\
\hline np & TW & 2.08 & 2.46 & 10.62 & 7.20 \\
\hline np+hyperons $(*)$ & TW & 1.89 & 2.24 & 9.46 & 8.44 \\
\hline $\mathrm{np}$ & $\mathrm{S} 3$ & 2.88 & 3.57 & 12.81 & 4.57 \\
\hline $\mathrm{np}+$ hyperons & S3 & 2.65 & 3.36 & 11.33 & 5.60 \\
\hline $\mathrm{np}$ & MA & 2.86 & 3.59 & 11.79 & 5.29 \\
\hline$n p+$ hyperons & MA & 2.76 & 3.49 & 11.00 & 5.93 \\
\hline
\end{tabular}

TABLE III: Hadronic star properties for the EOSs described in the text 


\section{THE IMPORTANCE OF THE REARRANGEMENT TERMS}

In what follows we concentrate on the role played by the rearrangement term in the different models. This term is very important and influences all properties of nuclear and neutron star matter since it changes the effective chemical potentials given in equations (24),(41) and (59).

In figure 10 we show the rearrangement term which arises in various situations. In figure 10 $\mathrm{k}$, it is shown the rearrangement terms which appear in symmetric nuclear matter (without the imposition of $\beta$ stability) for the TW and the two parametrizations of the BR scaled model within the density range considered in nuclear matter studies. The S3 parametrization produces a term which is much more negative than the TW model. The MA parametrization shows a rearrangement term which decreases even further. So, in this case the rearrangement term is more attractive within the BR-scaled models. Notice that in the S3 parametrization, if $y$ and $z$ were chosen as having the same value, only the scalar meson would contribute to the rearrangement term.

Please, notice that the scales of the figures mentioned next are all different. In figures 10b and 10, we show the influence of the temperature on the rearrangement term of the two BR scaled models. As the temperature affects very little the term in the MA parametrization, the same does not happen if the S3 parametrization is chosen. Moreover, the rearrangement term increases slightly as temperature increases within the MA and decreases quite a lot with the increase of temperature with the S3 choice.

In figure 10], the rearrangement term of the TW model is shown for symmetric nuclear matter, for a very asymmetric nuclear matter, with a proton fraction $y_{p}=0.1$ and for the equation of state where charge neutrality and $\beta$ stability is required either with only protons and neutrons or with hyperons as well. The same is shown in figures 10, and 10, for S3 and MA. While in the TW model the rearrangement term decreases with the asymmetry of the system and increases when the conditions of $\beta$ equilibrium and charge neutrality are enforced, the influence of the hyperons being very small, in the S3 all curves are very similar and in the MA the asymmetry almost does not interfere in the rearrangement term and the hyperons again play no role, but charge neutrality and $\beta$ equilibrium conditions modify the curve quite drastically.

In figures 10b and 10 one can see a comparison between the rearrangement term arising from the TW model and the ones obtained with the BR scaled models respectively for the case when only nucleons are considered and when hyperons are also included in the system. We have now opted to show a much wider density range so that the differences can be clearly seen. While the inclusion of hyperons makes the TW model rearrangement term increase slightly at densities of the order of $\simeq 2 \mathrm{fm}^{-3}$ as compared with the case when only nucleons are considered, at about the same density the MA rearrangement term starts to decrease. The S3 term also decreases more rapidly if hyperons are considered, but it starts at a density

of the order of $\simeq 1 \mathrm{fm}^{-3}$. At these very large densities other important features as the deconfinement to the quark matter is already present.

\section{CONCLUSIONS}

In the present work we have derived a formalism to incorporate temperature effects in the BR scaled model to make it useful in future heavy ion collision studies. We have also investigated the possibility of 
applying it to nuclear astrophysics by enforcing charge neutrality and $\beta$ equilibrium. We have compared our results with the more standard NL3 29] version of the NLWM in nuclear matter and with the GL 34] parametrization of the NLWM in neutron star matter. In both cases, the BR scaled model was also compared with another density dependent model, the TW [12]. It is worth pointing out that density dependent models are alternative approaches to describe hadronic matter without the usual non-linear terms necessary in the NLWM. Although in the low baryonic density regime all models and parametrizations used in this paper have EOSs with similar behavior, different scenarios show up when the density increases, specially, when the baryon octet is taken into account. The lagrangians with BR scaling are much simpler than the other model with density dependent couplings (TW) and they provide effective meson masses which decrease with the increase of the density, behavior which has already been confirmed by experiments [5, 6] and which is not present in all models where the meson masses are held fixed. We have also shown that the BR scaled model is a very good tool in describing neutron star properties. Of course a more systematic study can be done by including the delta [23, 32] and the strange 32, 42] mesons in the lagrangian density. Other possible choices for the meson-hyperon couplings should also be considered. More realistic hybrid stars with a deconfinement to the quark phase can now be obtained within the BR scaled model for the hadron phase. When the new EOSs are built and the stellar properties are obtained, the mass to radius constraints [18, 19] can be used as a probe to the new formalism. It is also important to say that the rearrangement term plays a central role in density dependent models and many of the system properties depend on its strength.It is crucial to assure the energy-momentum conservation and the thermodynamical consistency for density dependent models. In contrast to the pressure and compressibility which depends explicitly on the rearrangement term, in the energy density it cancels out. However, it still contributes to the binding energy through the chemical potentials. In figure 10 it was shown that the BR parametrizations yields more attractive rearrangement term as compared with the TW model.

We have checked that other possibilities for the parametrization of the BR scaled model given by

$$
\frac{g_{s}^{*}}{g_{s}}=\frac{1+x_{1} \rho / \rho_{0}}{1+x_{2} \rho / \rho_{0}}, \quad \frac{g_{v}^{*}}{g_{v}}=\frac{1+x_{3} \rho / \rho_{0}}{1+x_{4} \rho / \rho_{0}}, \frac{g_{\rho}^{*}}{g_{\rho}}=\frac{1+x_{5} \rho / \rho_{0}}{1+x_{6} \rho / \rho_{0}},
$$

also work. Nevertheless, one of the advantages of using the BR scaled models instead of the TW model is that they contain a much smaller number of parameters. With the choice given in (64) much of the beauty of the model would be lost, but it remains as a possible alternative. Finally, it is important to stress that a test to finite nuclei in order to obtain the correct value for the spin-orbit splitting strength is still necessary with the BR parameter sets used in this work.

\section{ACKNOWLEDGEMENTS}

This work was partially supported by CNPq (Brazil) and Capes (Brazil) under process BEX 1681/04-4. D.P.M. would like to thank the friendly atmosphere at the Reserch Centre for Theoretical Astrophysics, 
Sydney University, where this work was partially done.

[1] S.S. Avancini, L. Brito, D.P. Menezes and C. Providência, Phys. Rev. C70, 015203 (2004).

[2] F. Wilczek et al., Phys. Today August 10 (2002).

[3] T. Hatsuda and T. Kunihiro, Prog. Theor. Phys. 74, 765 (1985).

[4] G.E. Brown and M. Rho, Phys. Rev. Lett. 66, 2720 (1991).

[5] D. Trnka et al, CBELSA/TAPS Collaboration, nucl-ex/0504010

[6] M. Naruki et al. nucl-ex/0504016

[7] J. Adams et al., Phys. Rev. Lett. 92,092301 (2004); K. Suzuki et a., Phys. Rev. Lett. 92,072302 (2004); G.M. Huber et al., Phys. Rev C 68, 5019 (2001);G. Agakichiev et al., Phys. Lett. B 422, 405 (1998).

[8] W. Schön et al., Act. Phys. Pol. B 27, 2959 (1996).

[9] B. Friman and M. Rho, Nucl. Phys. A606, 303 (1996).

[10] C. Song, G.E. Brown, D. P. Min and M. Rho, Phys. Rev. C 56, 2244 (1997).

[11] B. Friman, M. Rho and C. Song, Phys. Rev. C 59, 3357 (1999).

[12] S. Typel and H. H. Wolter, Nucl. Phys. A656, 331 (1999).

[13] T. Gaitanos, M. Di Toro, S. Typel, V. Baran, C. Fuchs, V. Greco and H. H. Wolter, Nucl. Phys. A732, 24 (2004).

[14] S.S. Avancini, M.E. Bracco, M. Chiapparini and D.P. Menezes, Phys. Rev. C 67, 024301 (2003).

[15] S.S. Avancini, M.E. Bracco, M. Chiapparini and D.P. Menezes, J. Phys. G: Nuclear and Particle Physics 30, 27 (2004).

[16] D.P. Menezes and C. Providência - Phys. Rev C69, 045801 (2004).

[17] R.C. Tolman, Phys. Rev. 55, 364 (1939); J.R. Oppenheimer and G.M. Volkoff, Phys. Rev. 55, 374 (1939).

[18] J. Cottam, F. Paerels and M. Mendez, Nature 420, 51 (2002).

[19] D. Sanwal, G.G. Pavlov, V.E. Zavlin and M.A. Teter, Astrophys. J 574, L 61 (2002).

[20] G.F. Bignami, P.A. Caraveo,A. De Luca and S. Mereghetti, Nature 423, 725 (2003).

[21] R.X. Xu,H.G. Wang and G.J. Qiao, Chin. Phys. Lett. 20, 314 (2003).

[22] P.K. Panda, D.P. Menezes and C. Providência, Phys. Rev C69, 025207 (2004.

[23] D.P. Menezes and C. Providência - Phys. Rev C70, 058801 (2004).

[24] B.D. Serot and J.D. Walecka, Adv. Nucl. Phys. 16 (1986) 1.; J. Boguta and A. R. Bodmer, Nucl. Phys. A292, 413 (1977);

[25] A.M.S. Santos and D.P. Menezes, Phys. Rev. C69 (2004) 045803; Braz. J. Phys. 34, 833 (2004).

[26] C. Song, Phys. Rep. 347, 289 (2001).

[27] C. Song, D. P. Min and M. Rho, Phys. Lett. B 424, 226 (1998).

[28] D.P. Menezes and C. Providência, Phys. Rev. C 60, 024313 (1999).

[29] G. A. Lalazissis, J. König and P. Ring, Phys. Rev. C 55, 540 (1997).

[30] T. Hatsuda and H. Shiomi, Nucl. Phys. A590, 545c (1995).

[31] B.-A. Li, C.M. Ko and W. Bauer, Int. J. Mod. Phys. E 7, 147 (1997).

[32] F. Hofmann, C.M. Keil and H. Lenske, Phys. Rev. C 64, 025804 (2001).

[33] K. Sumiyoshi, H. Kuwabara, H. Toki, Nucl. Phys. A 581, 725 (1995).

[34] N. K. Glendenning, Compact Stars, Springer-Verlag, New-York, 2000.

[35] G. Hua, L.Bo and M. Di Toro, Phys. Rev. C 62, 035203 (2000).

[36] S. K. Ghosh, S. C. Phatak and P. K. Sahu, Z. Phys. A 352 (1995) 457.

[37] N. K. Glendenning and S. Moszkowski, Phys. Rev. Lett. 67, 2414 (1991). 


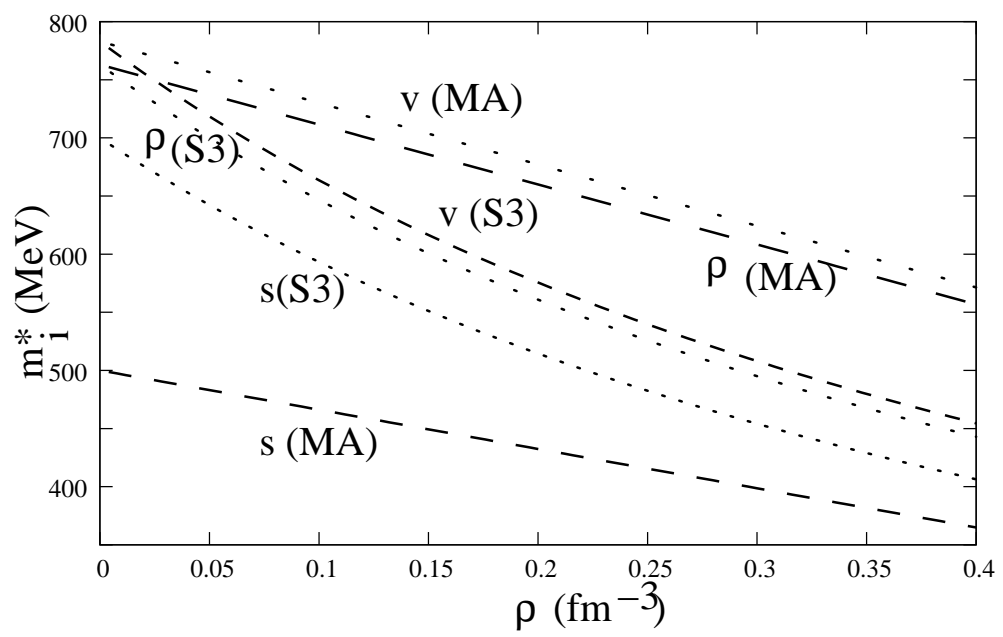

FIG. 1: Meson masses $m_{i}^{*}, i=s, v, \rho$ versus the baryonic density.

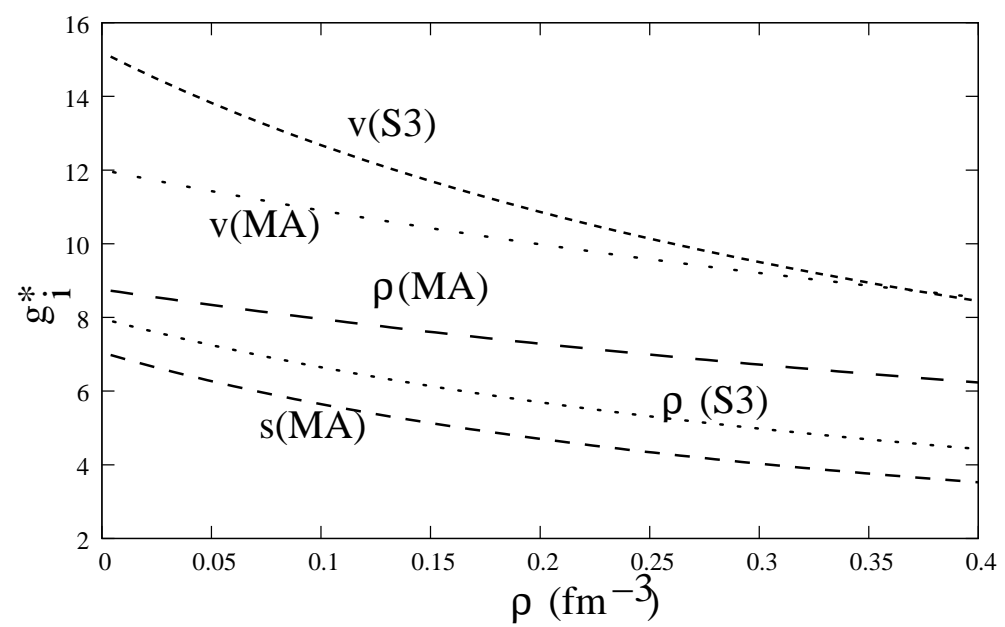

FIG. 2: Meson to baryon couplings $g_{i}^{* 2}, i=s, v, \rho$ in terms of the density.

[38] S.A. Moszkowski, Phys. Rev. D 9, 1613 (1974).

[39] N. K. Glendenning, Nucl. Phys. A 493, 521 (1989).

[40] N. K. Glendenning, Astrophys. J. 293, 470 (1985).

[41] A.L. Espíndola and D.P. Menezes, Phys. Rev. C 65, 045803 (2003).

[42] R. Cavangnoli and D.P. Menezes, Braz. J. Phys. (2005), in press, nucl-th/0506064 


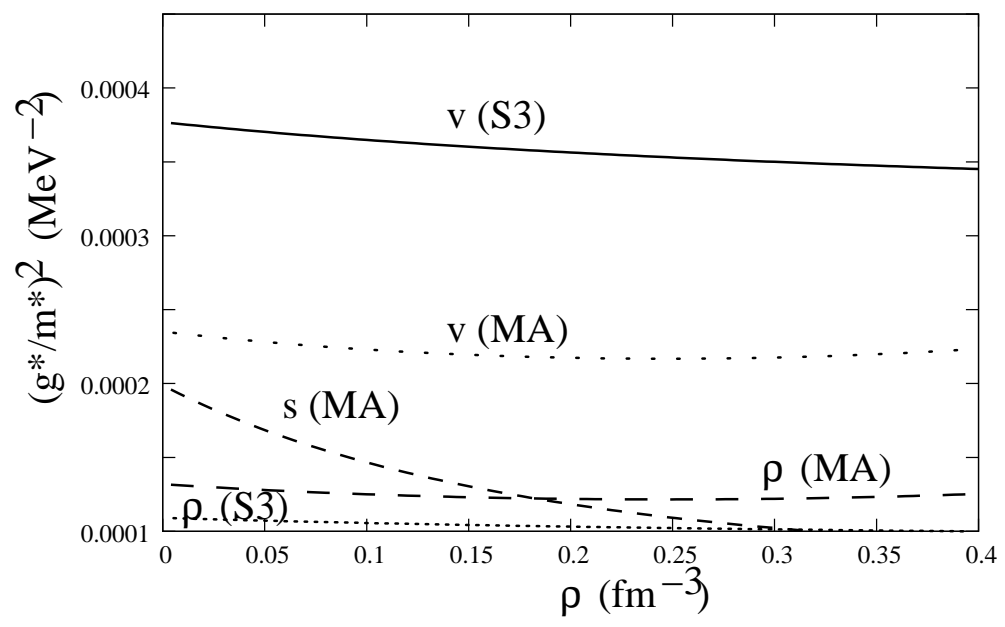

FIG. 3: Ratios $g_{i}^{* 2} / m_{i}^{* 2}, i=s, v, \rho$ as function of the density.
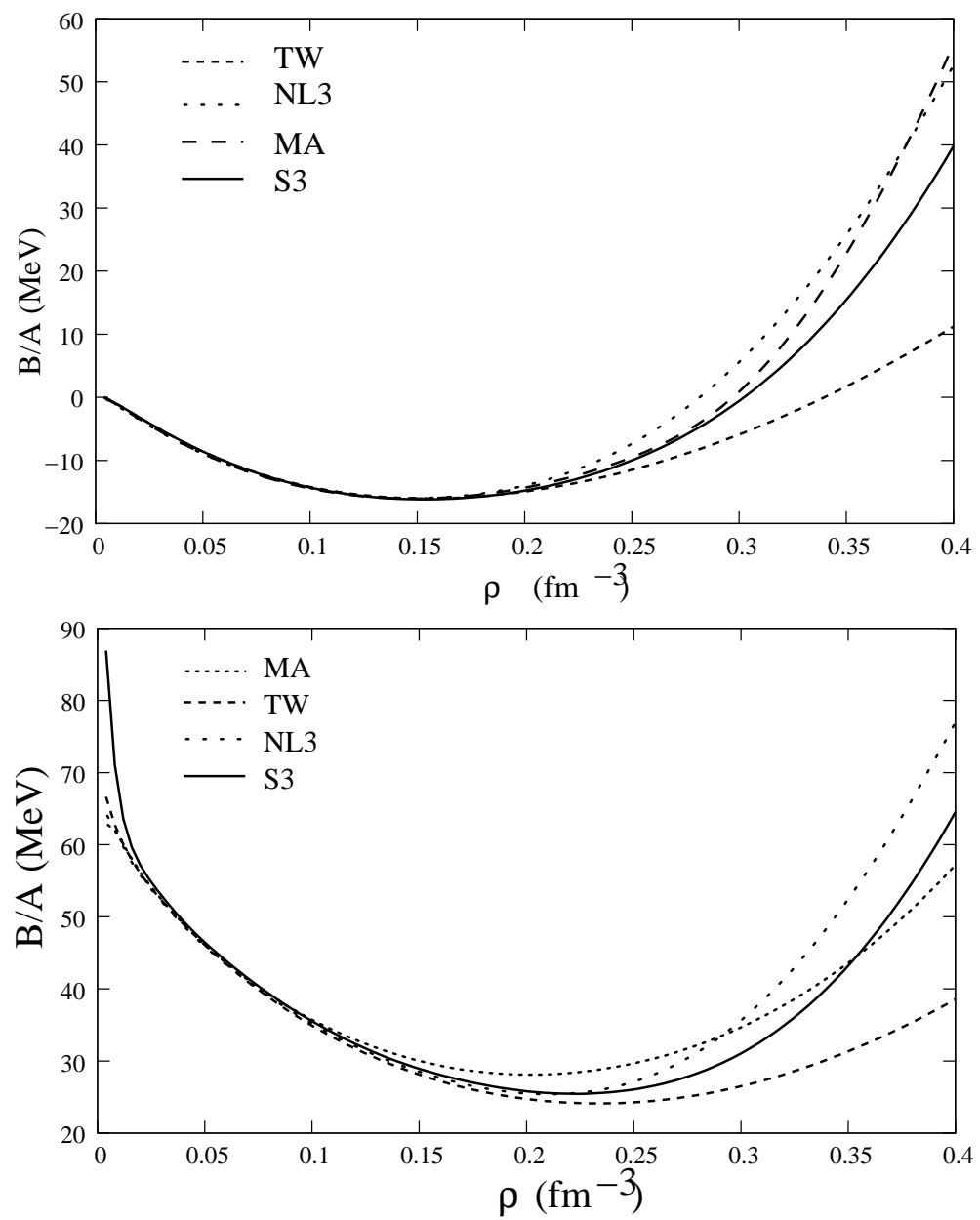

FIG. 4: Binding energy in terms of the baryon density for different models at $T=0$ (top figure) and $T=40 \mathrm{MeV}$ (bottom figure). 


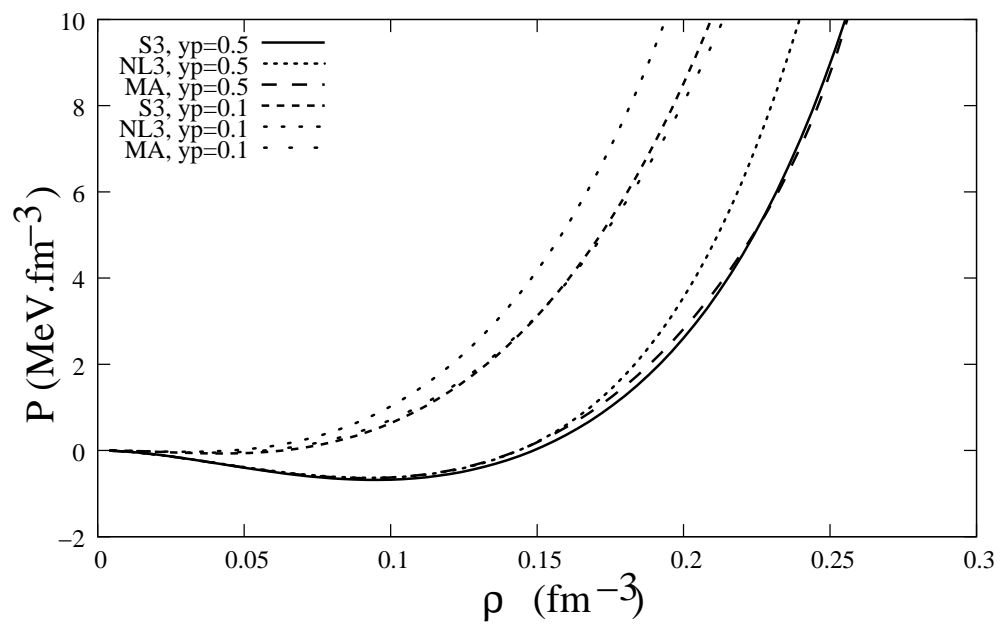

FIG. 5: Pressure in terms of the baryon density for different models at $T=0$ and two different proton fractions.

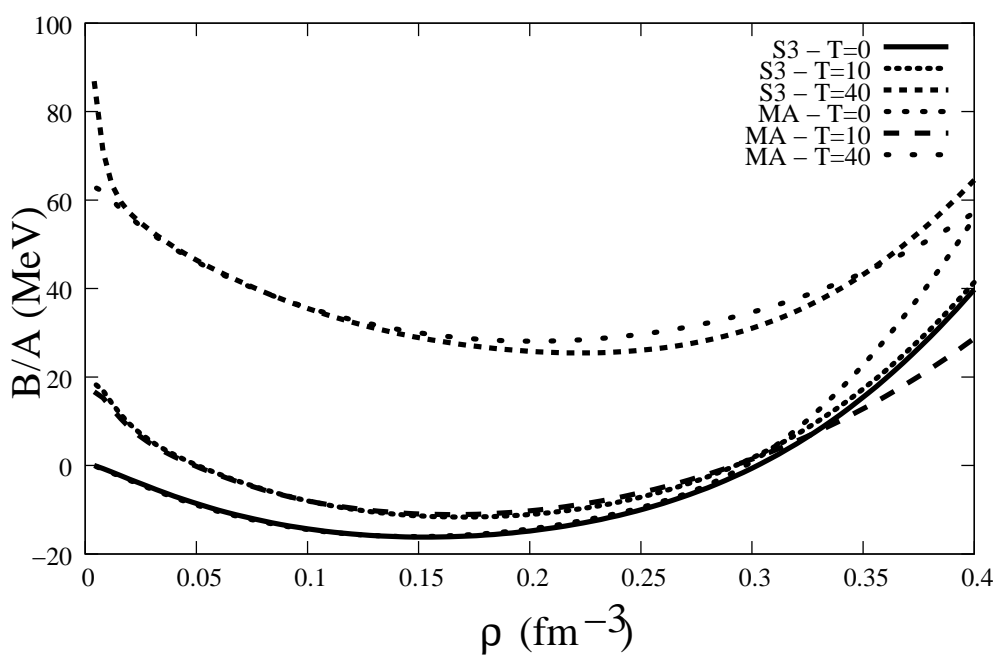

FIG. 6: Binding energy in terms of the baryon density for S3 and MA and different temperatures. 


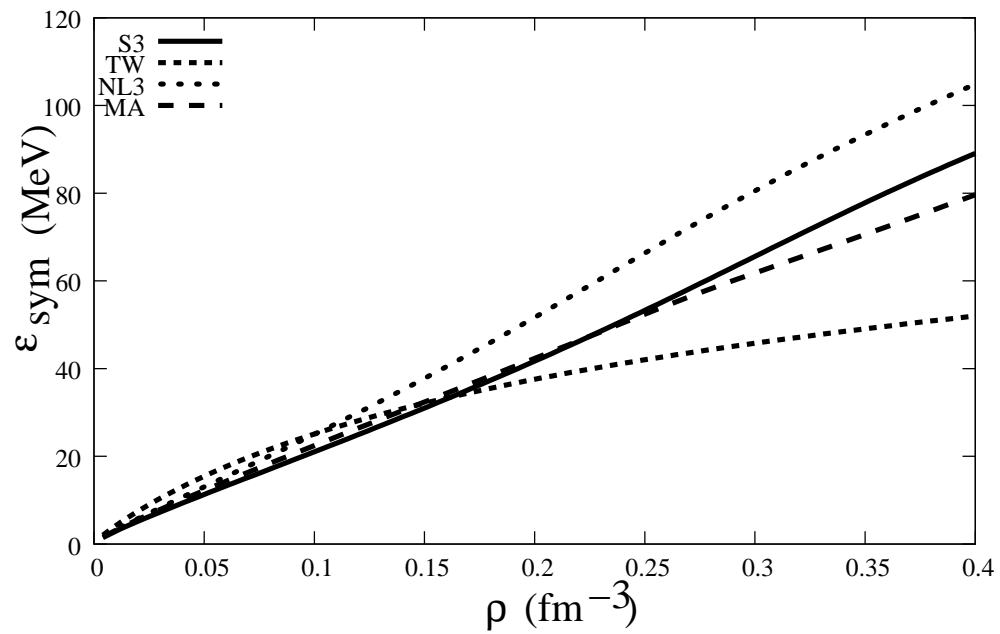

FIG. 7: Symmetry energy in terms of the baryon density for different models at $T=0$. 

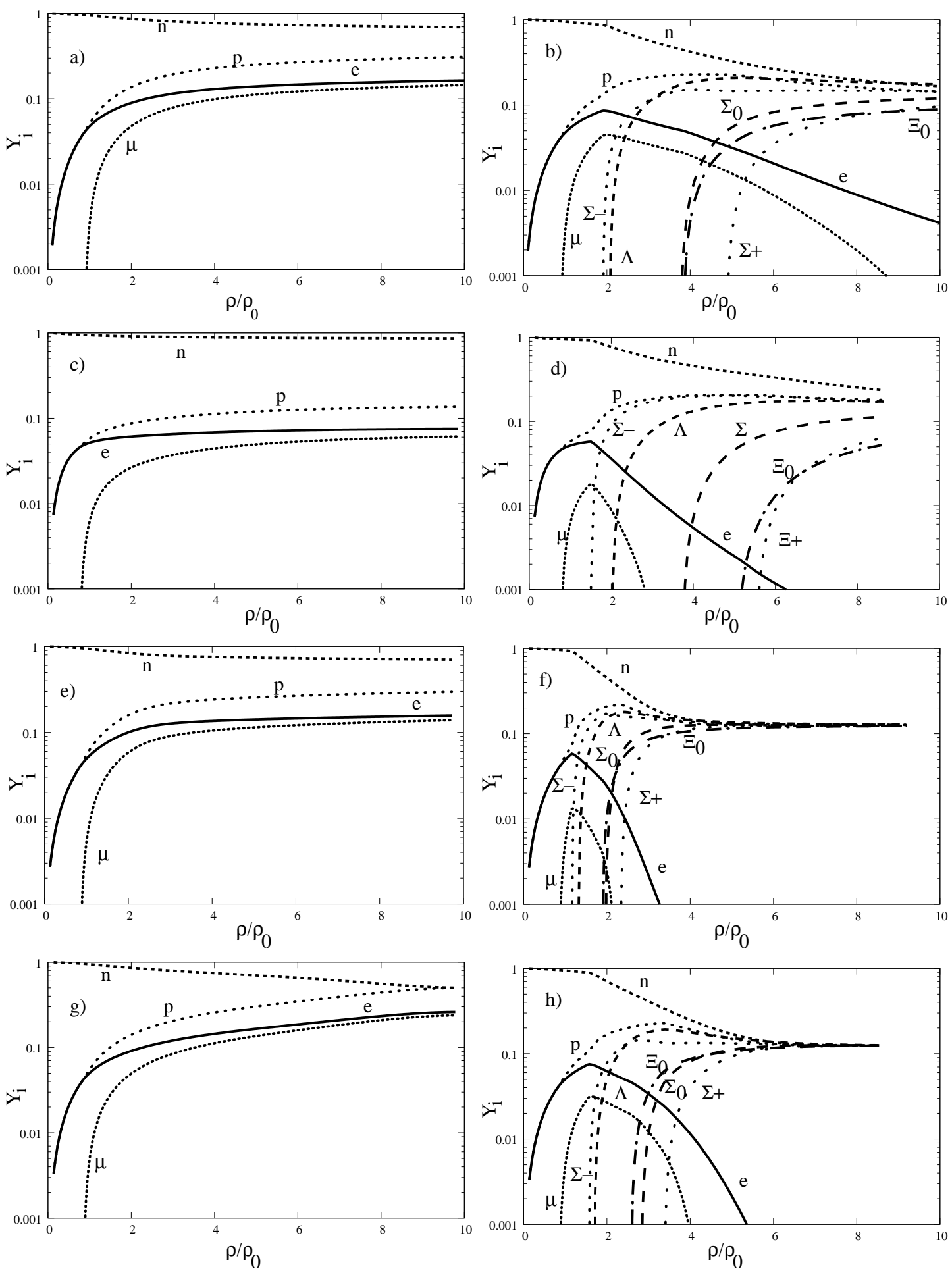

FIG. 8: Particle fractions for a) the non linear Walecka model with the GL parametrization with nucleons only, b) with 8 baryons, c) TW model with nucleons only, d) with 8 baryons, e) BR model with parameter sets S3 and nucleons only f) S3 and 8 baryons, g)MA and nucleons only, h) MA and 8 baryons. 

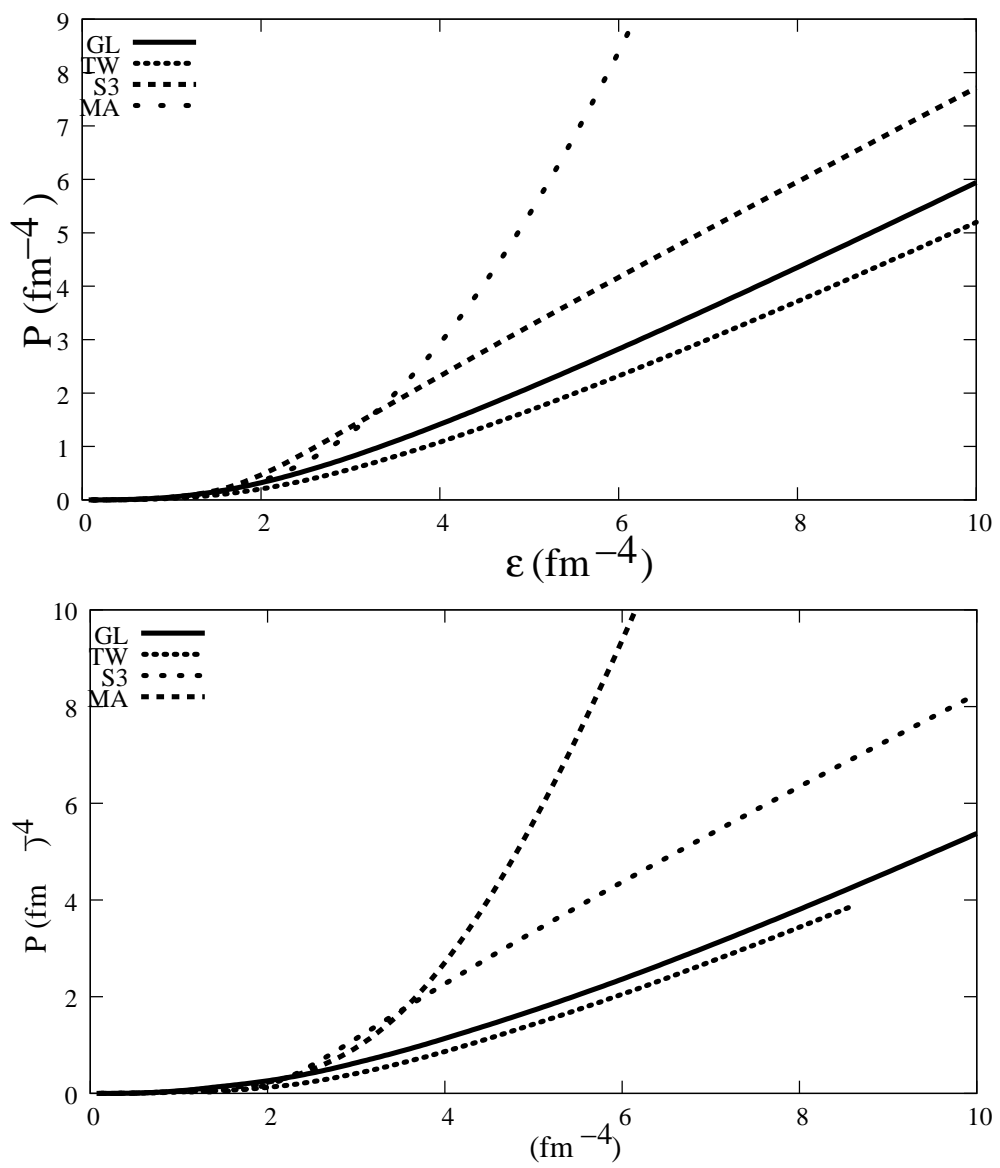

FIG. 9: EOS for the models discussed in the present work with nucleons only (top figure) and 8 baryons (bottom figure). 

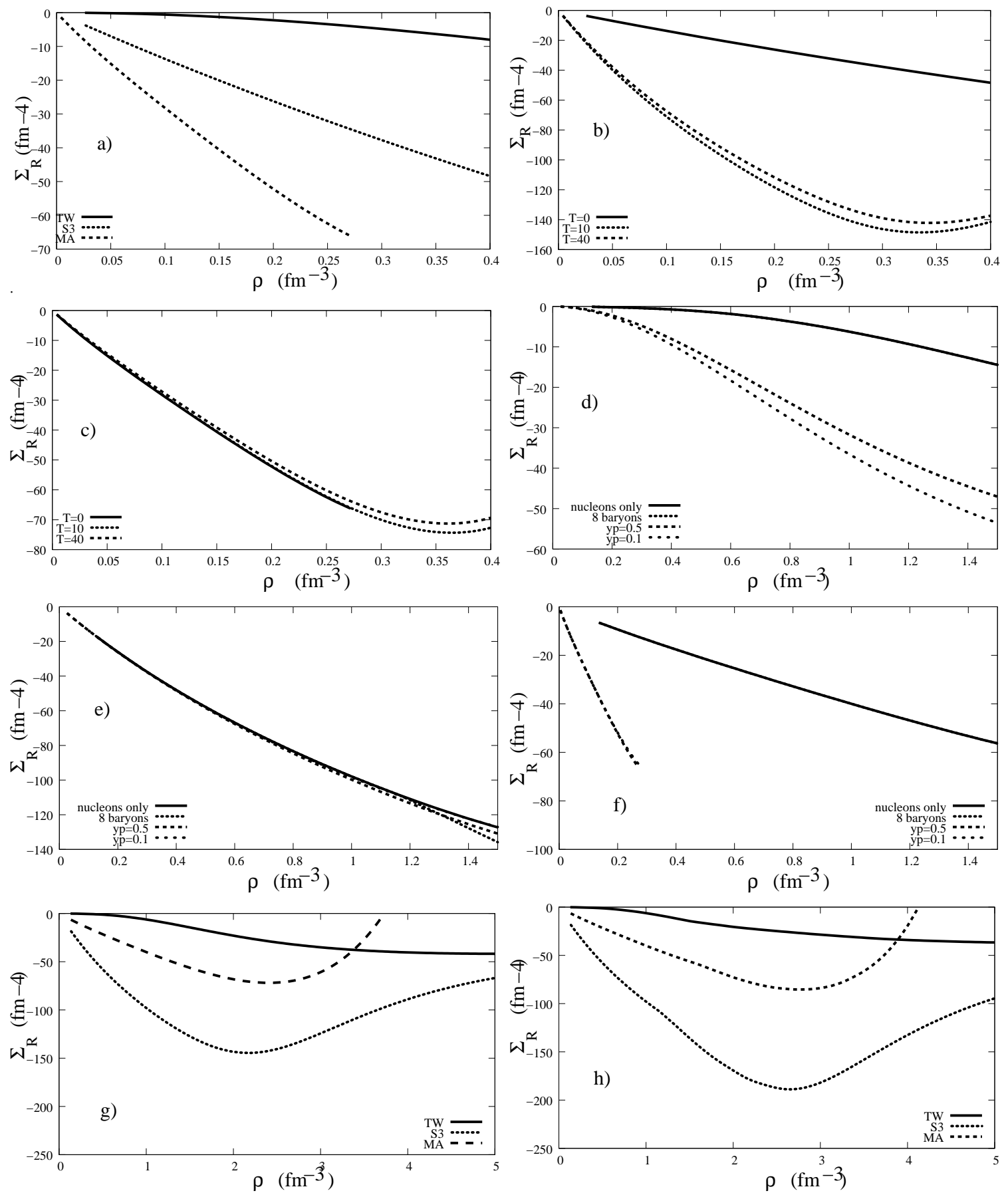

FIG. 10: Rearrangement terms calculated in different situations: a)different models in symmetric nuclear matter, b) S3 and symmetric nuclear matter for different temperatures, c) MA and symmetric nuclear matter for different temperatures, d) TW, e) S3, f) MA, g) nucleons only, h) 8baryons. 